\title{
Clinical course and outcome of differentiated thyroid cancer patients with pregnancy after diagnosis of distant metastasis
}

Haruhiko Yamazaki ( $\square$ paruo0413@gmail.com )

Ito Hospital https://orcid.org/0000-0002-6640-3529

Kiminori Sugino

Ito Hospital

Jaeduk Yoshimura Noh

Ito Hospital

Ryohei Katoh

Ito Hospital

Kenichi Matsuzu

Ito Hospital

Chie Masaki

Ito Hospital

Junko Akaishi

Ito Hospital

Kiyomi Yamada Hames

Ito Hospital

Chisato Tomoda

Ito Hospital

Akifumi Suzuki

Ito Hospital

Keiko Ohkuwa

Ito Hospital

Wataru Kitagawa

Ito Hospital

Mitsuji Nagahama

Ito Hospital

\section{Yasushi Rino}

Yokohama City University Hospital: Yokohama Shiritsu Daigaku Fuzoku Byoin

Koichi Ito

Ito Hospital 


\section{Research Article}

Keywords: Differentiated thyroid cancer, distant metastasis, pregnancy, prognosis

Posted Date: August 17th, 2021

DOl: https://doi.org/10.21203/rs.3.rs-800597/v1

License: (c) (i) This work is licensed under a Creative Commons Attribution 4.0 International License.

Read Full License

Version of Record: A version of this preprint was published at Endocrine on January 22nd, 2022. See the published version at https://doi.org/10.1007/s12020-021-02969-7. 


\section{Abstract \\ Purpose}

There is no sufficient data about the clinical course and outcome in thyroid cancer patients who become pregnant after diagnosis of distant metastasis (DM). The current study was conducted to collect information regarding the clinical and reproductive characteristics, and outcomes in thyroid cancer patients who became pregnant after being diagnosed with DM.

\section{Methods}

Records of 125 differentiated thyroid cancer (DTC) patients with age $\leq 45$ years at DM diagnosis who had visited Ito Hospital from January 2005 to June 2021 were retrospectively reviewed. Among those 125 patients, 28 who became pregnant after DM diagnosis were classified as pregnant group, and the remained 97 patients were classified as comparator group.

\section{Results}

In pregnant group, the median age at malignancy diagnosis, DM diagnosis, and first pregnancy after DM diagnosis was 25 years (range, 4-41 years), 27 years (range, 11-41 years), and 32 years (range, 25-45 years), respectively. Fifty-five pregnancies and 40 live births were reported. Three patients had live births by embryo transfer. Other pregnancy outcomes were miscarriage $(n=14)$ and induced abortion $(n=1)$. No one died during the follow-up period in this study. The 10-year progression free survival (PFS) rates of pregnant and comparator group were $92.1 \%$ and $74.4 \%$, respectively.

\section{Conclusion}

DTC patients who became pregnant after DM diagnosis had good survival. Our results add to the information required for counseling thyroid cancer patients who have concerns about their fertility in the future.

\section{Introduction}

Thyroid cancer is the most common type of endocrine-related malignancy [1]. Papillary thyroid carcinoma and follicular thyroid carcinoma are types of differentiated thyroid cancer (DTC) that account for $>95 \%$ of all thyroid carcinomas [2]. The prognosis of DTC is good, with a disease-specific survival rate $>90 \%$ [3]. However, some DTC patients experience recurrence, and the prognosis of patients with unresectable, advanced DTC remains poor [4]. Conditions involving distant metastasis (DM) of the lungs or bone are considered to be incurable, and radioactive iodine (RAI) or multi kinase inhibitor is treatment options in patients with these conditions $[2,5]$. 
The staging system for thyroid cancer is unique, and age is considered an important prognostic factor. As per the American Joint Committee on Cancer (AJCC) Eighth Edition staging system of thyroid cancer, age of 55 years is used as a stratification value [6]. DTC patients with age $<55$ years and DM are diagnosed

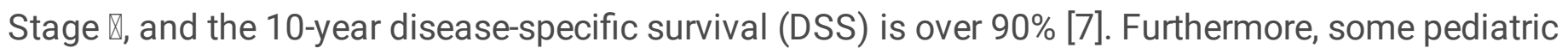
thyroid cancer patients have good prognosis even though they had DM at the time of diagnosis of malignancy [8]. Young thyroid cancer patients with DM survive longer than those with other malignancies, and some patients may wish to become pregnant.

Thyroid cancer is the second most common pregnancy associated with cancer [9]. Pregnancy-related hormones, such as estrogen and human chorionic gonadotropin, may favor the growth, progression, and spread of thyroid tumors $[10,11]$. However, to our knowledge, there is no evidence showing that pregnancy worsens the prognosis of thyroid cancer $[12,13]$. Xi et al. revealed that pregnancy does not affect the prognoses in DTC patients with lung metastasis [13].

In recent times, post-treatment life events, such as pregnancy and childbirth, have been considered an essential part of treatment planning for adolescent cancer patients because advances in cancer therapy have substantially increased the long-term survival rate of adolescent and young adult cancer survivors [14]. However, this issue remains unclear because there is insufficient data, especially in thyroid cancer patients with DM. Thus, we conducted an observational study to collect information regarding the clinical and reproductive characteristics, and outcomes in thyroid cancer patients who became pregnant after being diagnosed with DM. Furthermore, we compared the prognosis between those patients and comparator group.

\section{Materials And Methods}

\section{Subjects}

We screened records of 952 thyroid cancer patients with DM who had visited Ito Hospital from January 2005 to June 2021 were retrospectively reviewed. The inclusion criteria were female, age $\leq 45$ years at DM diagnosis, differentiated thyroid cancer confirmed using surgical specimens. The exclusion criteria were male, age > 45 years at DM diagnosis, medullary thyroid cancer, poorly differentiated thyroid cancer, anaplastic thyroid cancer, previous history of other primary malignancy, and not meeting the criteria of DM. Since the success rate of fresh autologous treatment for women aged $\geq 45$ years was very low (< $1 \%$ ) and this age may be considered to be upper limitation of age for assisted reproductive technology (ART), we collected information of patients with age $\leq 45$ years [15]. Finally, 125 patients were enrolled. Among those 125 patients, 28 who became pregnant after DM diagnosis were classified as pregnant group, and the remained 97 patients were classified as comparator group. Enrollment and participation flow diagram was shown in Fig. 1. Information regarding the patients' baseline and reproductive characteristics, laboratory data, RAI treatment history, disease progression, and death were subsequently collected for the analyses. Regular palpation examinations, and measurements of thyroid function and thyroglobulin (Tg) were performed every 3-6 months. A systemic survey such as computed tomography 
(CT) or positron emission tomography (PET)/CT was performed every 12 months or when clinicians considered disease progression (e.g., elevation of serum thyroglobulin, appearance bone pain). During pregnancy, measurements of thyroid function and thyroglobulin were performed every 2-3 months to control thyroid stimulating hormone (TSH) levels in optima range, and systemic survey was not performed. Fertility was assessed using the data collected using a self-administered questionnaire and medical records. The protocol employed in this retrospective study was approved by the Institutional Review Board of Ito Hospital (approval no. 336).

\section{Definitions}

DM was diagnosed based on CT, PET/CT, therapeutic ${ }^{131} \mathrm{I}$-whole body-scan ( ${ }^{131} \mathrm{I}-\mathrm{WBS}$ ), and serum $\mathrm{Tg}$ levels [12]. Dosage of ${ }^{131} \mathrm{I}$ of $25 \mathrm{mCi}$ or higher was considered as therapeutic doses. Overall survival (OS) was calculated as the duration from the point of diagnosis of malignancy to the date of death from any cause. Progression-free survival (PFS) was calculated as the duration from the point of diagnosis of DM to disease progression or the date of death from any cause. Disease progression was defined as $\geq 20 \%$ increase in DM lesion volumes or the appearance of new lesions.

\section{Statistical analysis}

All the statistical analyses were performed using EZR (Saitama Medical Center, Jichi Medical University, Saitama, Japan), a graphical user interface for R (The R Foundation for Statistical Computing, Vienna, Austria) [16]. Categorical and continuous variables were compared using Fisher's exact test and Chi square test, and Mann-Whitney U test, respectively. PFS curves were constructed using the Kaplan-Meier method. Cox proportional hazards model was used to determine factors associated with PFS. A $p$ value of $<0.05$ was considered to indicate statistical significance.

\section{Results}

\section{Baseline Characteristics}

The baseline clinicopathological characteristics of the patients in pregnant and comparator group are shown in Table 1. In pregnant group, the median age at malignancy diagnosis and DM diagnosis was 25 years (range, $4-41$ years) and 27 years (range, $11-41$ years), respectively. Fifteen patients (54\%) had DM at the time of malignancy diagnosis. Two patients had lung and bone metastases and the remaining 26 patients had only lung metastasis. The median $\mathrm{Tg}$ concentration measured at the time of DM diagnosis was $109.4 \mathrm{ng} / \mathrm{dL}$ (range, $0.5-7000 \mathrm{ng} / \mathrm{dL}$ ). The median cumulative activity of ${ }^{131}$ I was $200 \mathrm{mCi}$ (range, 0-600). Among the 23 patients whose follow-up Tg data were available, 21 (91\%) had lower Tg concentration at the point of first pregnancy after DM diagnosis than at the point of DM diagnosis; however, this difference was not statistically significant $(p=0.151)$. There were significant differences in age at diagnosis of malignancy, age at diagnosis of $\mathrm{DM}$, and cumulative ${ }^{131} \mathrm{I}$ activity between two groups (Table 1). 
Table 1

Baseline clinicopathological characteristics

\begin{tabular}{|c|c|c|c|}
\hline & $\begin{array}{l}\text { Pregnant } \\
\text { group } \\
(n=28)\end{array}$ & $\begin{array}{l}\text { Comparator } \\
\text { group } \\
(n=97)\end{array}$ & $\begin{array}{l}\mathrm{p} \\
\text { value }\end{array}$ \\
\hline Age at malignancy diagnosis (years) & $25(4-41)$ & $31(6-45)$ & 0.005 \\
\hline Age at distant metastasis diagnosis (years) & $27(11-41)$ & $34(6-45)$ & 0.009 \\
\hline Histology, n (\%) & & & 0.532 \\
\hline Papillary & $23(82)$ & $85(88)$ & \\
\hline Follicular & $5(18)$ & $12(12)$ & \\
\hline Tumor-node-metastasis stage, n (\%) & & & 0.354 \\
\hline \multicolumn{4}{|l|}{$\mathrm{T}$} \\
\hline T1-2 & $11(39)$ & $28(29)$ & \\
\hline T3-4 & $11(39)$ & $53(55)$ & \\
\hline TX & $6(22)$ & $16(16)$ & \\
\hline $\mathrm{N}$ & & & 0.551 \\
\hline NO & $2(7)$ & $15(15)$ & \\
\hline N1 & $20(71)$ & $65(67)$ & \\
\hline NX & $6(22)$ & $17(18)$ & \\
\hline M & & & 0.070 \\
\hline MO & $13(46)$ & $27(28)$ & \\
\hline M1 & $15(54)$ & $70(72)$ & \\
\hline Metastatic site, $\mathrm{n}(\%)$ & & & 0.792 \\
\hline Lung & $28(100)$ & $90(93)$ & \\
\hline Bone & $2(7)$ & $11(11)$ & \\
\hline Brain & 0 & $1(1)$ & \\
\hline $\begin{array}{l}\text { Thyroglobulin concentration at diagnosis of distant } \\
\text { metastasis }\end{array}$ & $\begin{array}{l}109.4(0.5- \\
7000.0)\end{array}$ & $\begin{array}{l}80.0(0.2- \\
4800.0)\end{array}$ & 0.997 \\
\hline Anti-thyroglobulin antibody, n (\%) & & & 0.059 \\
\hline
\end{tabular}

Numbers are presented as median (range). ※Thyroglobulin concentrations at diagnosis of distant metastasis were unknown in five and 21 patients in pregnancy and comparator group, respectively. 


\begin{tabular}{|llll|}
\hline & $\begin{array}{l}\text { Pregnant } \\
\text { group } \\
(\mathbf{n = 2 8})\end{array}$ & $\begin{array}{l}\text { Comparator } \\
\text { group } \\
(\mathbf{n = 9 7 )}\end{array}$ & $\begin{array}{l}\mathbf{p} \\
\text { value }\end{array}$ \\
\hline Positive & $1(4)$ & $21(22)$ \\
\hline Negative & $19(68)$ & $51(51)$ & \\
\hline Unknown & $8(28)$ & $25(26)$ & 0.023 \\
\hline Cumulative ${ }^{131}$ I activity (mCi) & $200(0-600)$ & $130(0-1059)$ & 0.820 \\
\hline Multiple ${ }^{131}$ I treatment, $n(\%)$ & $18(64)$ & $57(59)$ & \\
\hline $\begin{array}{l}\text { Numbers are presented as median (range). ※Thyroglobulin concentrations at diagnosis of distant } \\
\text { metastasis were unknown in five and 21 patients in pregnancy and comparator group, respectively. }\end{array}$ \\
\hline
\end{tabular}

\section{Reproductive characteristics}

The reproductive characteristics are shown in Table 2. The median age at first pregnancy after DM diagnosis was 32 years (range, 25-45 years). Of the 28 patients, 10 (36\%) had been pregnant at least once before they were diagnosed with DM. The median duration between the first pregnancy after DM diagnosis and the point of DM diagnosis was 4.8 years (range, $0.3-18.9$ years), and the median duration between the first pregnancy after DM diagnosis and the last ${ }^{131}$ I treatment was 2.1 years (range, $0.5-14.5$ years). The median TSH level measured at first pregnancy was $1.03 \mu \mathrm{lU} / \mathrm{mL}$ (range, $0.5-14.5 \mu \mathrm{lU} / \mathrm{mL}$ ). Fifty-five pregnancies and 40 live births were reported. Three patients had live births by embryo transfer. Among 40 live births, the incidence of premature birth was $5.0 \%(n=2)$, external abnormally was $5.0 \%$ ( $n$ $=2)$, small for dates was $2.5 \%(n=1)$, and heavy for dates was $2.5 \%(n=1)$, respectively. Other pregnancy outcomes were miscarriage $(n=14)$ and induced abortion $(n=1)$. Eight patients experienced miscarriage, and 3 of 8 reported multiple miscarriages. 
Table 2

Reproductive characteristics

$n=28$

Age at first pregnancy after distant metastasis diagnosis (years)

$32(25-45)$

Ever been pregnant before diagnosis of distant metastasis, $\mathrm{n}(\%)$

Yes

No

TSH level at first pregnancy after distant metastasis diagnosis $(\mu \mathrm{lU} / \mathrm{mL})$

$1.03(0.01-6.10)$

Time to first pregnancy after distant metastasis diagnosis (years)

$4.8(0.3-18.9)$

Time to first pregnancy after last radioactive iodine treatment (years)

$2.1(0.5-14.5)$

Cumulative ${ }^{131}$ I activity before first pregnancy $(\mathrm{mCi})$

$200(130-600)$

Multiple ${ }^{131}$ I treatment before pregnancy, $\mathrm{n}(\%)$

$17(61)$

No. of pregnancies, $n$

55

Live birth, $\mathrm{n}$

40

Women reporting miscarriage, $\mathrm{n}$

14

Induced abortion, $\mathrm{n}$

1

TSH, thyroid stimulating hormone.

Numbers are presented as median (range). Among 40 live births, the incidence of premature birth was $5.0 \%(n=2)$, external abnormally was $5.0 \%(n=2)$, small for dates was $2.5 \%(n=1)$, and heavy for dates was $2.5 \%(n=1)$, respectively. Eight patients experienced miscarriage, and 3 of 8 reported multiple miscarriages.

\section{Survival and prognosis}

The median follow-up time was 10.9 years (range, 4.2-32.5 years) in pregnant group and 6.6 years (range, 0.1-36.0 years) in comparator group. No one died during the follow-up period in this study. The results of the univariate and multivariate analyses of PFS in all 125 patients with DM are summarized in Table 3. An age of $>34$ years (the median age of all 125 patients) $(p=0.007)$, multiple ${ }^{131} \mid$ treatment $(p=$ $0.039)$, presence of distant metastasis other than lung $(p=0.009)$, and non-pregnancy $(p=0.018)$ were related to poor PFS in the univariate analysis. The 10-year PFS rates of pregnant and comparator group were $92.1 \%$ and $74.4 \%$, respectively (Fig. 1). We performed multivariate analysis by including the factor of age, histology, multiple ${ }^{131}$ I treatment, distant metastatic site, and pregnancy. The multivariate analysis showed that multiple ${ }^{131}$ I treatment was independent prognostic factor for PFS ( $\left.p=0.046\right)$, and the history of pregnancy after DM diagnosis did not have negative effect on PFS. Among 28 patients in pregnant group, three patients exhibited disease progression during the follow-up period, and all the 
events occurred after pregnancy. One patient developed bilateral lower extremity paralysis at 37 week of gestation because of bone metastasis progression. She underwent emergent and cesarean section, and lenvatinib was started after wound healing. 
Table 3

Univariate and multivariate analysis of progression free survival

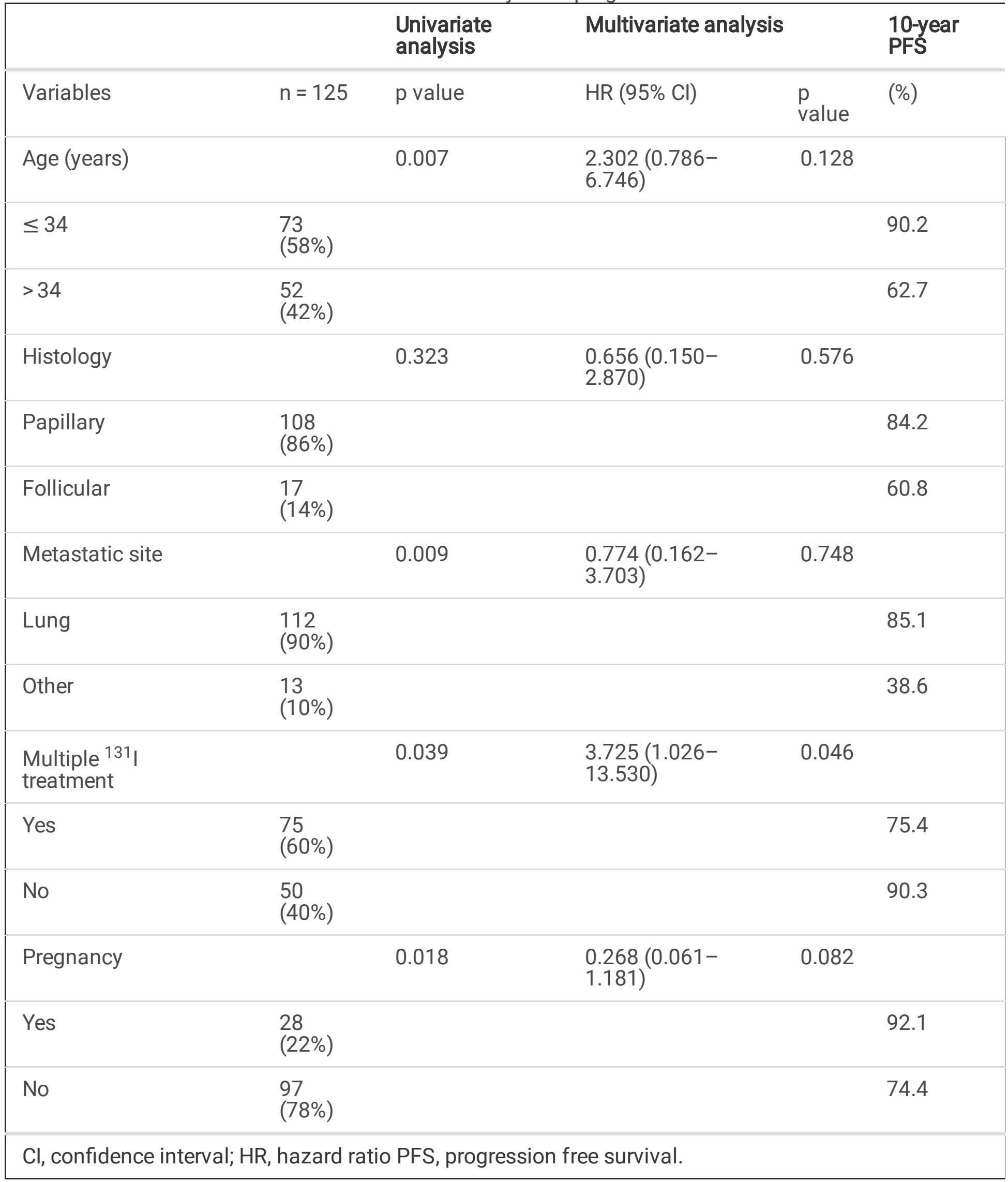

\section{Discussion}


In this study, we investigated the clinical characteristics and outcomes in thyroid cancer patients who became pregnant after they were diagnosed with DM. All the 28 patients enrolled in the present study had very good prognosis; no one died in the follow-up period although the patients had DM. In addition, most patients whose 10 -year PFS rate was $92.1 \%$ did not develop disease progression after pregnancy.

Age is a critical factor that influences the outcome in patients with well-DTC. The stratification age of 55 years is now used in the AJCC eighth edition staging system of thyroid cancer, and patients aged $<55$ years are diagnosed to have Stage I or II disease. Nixon et al. reported that the 10-year DSS rates of Stage I and II patients were $99.5 \%$ and $94.7 \%$, respectively [7]. First, DTC patients with DM are generally treated with RAI. The response rate for lung metastasis showing iodine accumulation is estimated to be $17 \%$ for complete response, $44 \%$ for partial response, $33 \%$ for stable disease, and $6 \%$ for progressive disease in the Japanese revised clinical practice guidelines on the management of thyroid tumors [17]. The 10-year survival rate of patients with lung metastasis showing iodine accumulation was good, at $87 \%$, and response to therapy was excellent, especially in young patients $[18,19]$. This study included only female patients who became pregnant after being diagnosed with DM; all 28 patients were aged $<55$ years at the time of DM diagnosis. Moreover, all the patients had lung metastasis. No one died during the follow-up period with a median duration of 15.5 years from the point of DM diagnosis, and the prognosis was considered very well, as in previous reports.

For DTC thyroid cancer patients, the effect of RAI on the reproductive function is an important consideration. Sawka et al. indicated that RAI treatment for DTC was generally not associated with a significantly increased risk of long-term infertility, miscarriage, induced abortions, stillbirths, or offspring neonatal mortality, or congenital defects [19]. Furthermore, Nies et al. reported that RAI treatment during childhood did not appear to impact the reproductive characteristics in female DTC patients [20]. In contrast, Ko et al. reported that patients treated with RAI had a lower successful delivery rate, particularly those aged 25-34 years as compared to those who did not undergo RAI [21]. In addition, Wu et al. revealed that the median time to the first live birth after DTC diagnosis was prolonged in women who received RAI than in those who did not [22]. Although it is controversial whether RAl affects the reproductive outcomes, RAI probably affects the timing of considering pregnancy in thyroid cancer patients. In Japanese patients with two or more unexplained consecutive miscarriages, the live birth rates ranged from $58.1-78.1 \%$ and were influenced by the maternal age [23]. As per other Japanese data, 33\% of the women did not reach delivery (mean maternal age 33.1 years) [24]. The birth rate of the patients included in this study was $73 \%$ (40/55), similar to that reported for other Japanese populations. Therefore, the previous RAl treatment possibly did not affect the fertility in our subjects.

During pregnancy, iodine deficiency, the TSH receptor-stimulating effect of human chorionic gonadotropin, and high estrogen levels are believed to promote growth of benign and malignant nodules. Previous studies have shown no differences in the survival, recurrence, or death between pregnancyassociated thyroid cancer and cancer in age-matched non-pregnant women [10] However, a more recent study reported that persistence/recurrence of DTC is significantly higher in pregnant patients, suggesting that pregnancy could exert a negative prognostic role in DTC patients, and more careful follow-up is 
needed in patients who are diagnosed with DTC during pregnancy or shortly thereafter [25]. Xi et al. reported that the 10 -year PFS and OS rates were $63.22 \%$ and $85.77 \%$, respectively, in the pregnancy group $(n=37)$ versus $58.13 \%$ and $81.95 \%$, respectively, in the non-pregnancy group $(n=87)$; thus, pregnancy does not affect the DTC prognoses in patients with lung metastasis [13]. In this study, the 10-year PFS rate $(92.1 \%)$ was better than that in a previous study. Young DTC patients may have good prognosis although they have DM. However, some DTC patients exhibited disease progression; therefore, we need to perform careful follow-up in patients with DM after pregnancy. Furthermore, one patient in this study started lenvatinib, a multi kinase inhibitor that is adapted for progressive and RAl-resistant DTC, after childbirth. In the future, for patients whose disease conditions were concerning, such as those considered for multi kinase inhibitor treatment, physicians need to be careful about recommending pregnancy.

Oncofertility care for female cancer survivors can include embryo/oocyte cryopreservation, ovarian tissue cryopreservation, and gonadotropin-releasing hormone agonist therapy. The Japan Agency for Medical Research and Development reports that $>1000$ embryos or oocytes and $>100$ ovarian tissue samples were cryopreserved for cancer patients between January 2011 and December 2015 [26]. Takahashi et al. analyzed the data of 67 Japanese women aged $<43$ years who underwent oncofertility care. The study indicated that as spontaneous pregnancies were more common than ART pregnancies, pregnancy via not only ART, but also via the non-ART method is a viable option for young cancer survivors [14]. In our study, 40 live births were reported, and three patients had live births from pregnancies induce via embryo transfer. The timing of fertility preservation discussion may be different for each type of cancer, and the previous report included only one thyroid cancer patient. DTC patients have longer survival than those with other malignancies even in the presence of DM; thus, some patients may desire to become pregnant by using ART.

The present study has certain limitations. Firstly, this was a single-center retrospective investigation that included a relatively small number of patients. Secondly, there was a degree of selection bias in this study because patients with better physical condition and stable disease are more likely to decide to become pregnant after being diagnosed with DM. Finally, since systemic survey could not be performed during pregnancy, it was uncertain whether disease progression truly occurred after becoming pregnant. Despite these limitations, we believe that our results are important because to our knowledge, this is the first detailed report on the reproductive and clinical characteristics of DTC patients who became pregnant after being diagnosed with DM. Our study may help in making a decision regarding whether pregnancy is advisable for patients and physicians in the future.

In conclusion, the DTC patients who became pregnant after DM diagnosis had good survival. However, some patients exhibited disease progression of DM after pregnancy, and physicians need to perform careful follow-up in these patients. Furthermore, for patients whose disease conditions are concerning, the patients and physicians need to discuss the decision of becoming pregnant. We believe that our results add to the information required for counseling thyroid cancer patients who have concerns about their fertility in the future. 


\section{Declarations}

\section{Acknowledgements}

We thank Enago (https://www.enago.jp/) for editing this manuscript.

\section{Funding}

No specific grant was received from any funding agency in the public, commercial, or not-for-profit sector for the publication of this report.

\section{Conflicts of interest}

The authors declare that they have no conflict of interest.

\section{Availability of data and material}

Not applicable.

\section{Code availability}

Not applicable.

\section{Ethics approval}

All procedures performed in studies involving human participants were in accordance with the ethical standards of the Ethics Committee of Ito Hospital (approval number 336) and with the 1964 Helsinki declaration and its later amendments or comparable ethical standards.

\section{Informed consents}

Informed consent was obtained from all individual participants included in the study.

\section{References}

1. Macerola E, Poma AM, Vignali P, Basolo A, Ugolini C, Torregrossa L, Santini F, Basolo F: Molecular Genetics of Follicular-Derived Thyroid Cancer. Cancers (Basel) 13:1139 (2021)

2. Yamazaki H, Iwasaki H, Takasaki H, Suganuma N, Sakai R, Masudo K, Nakayama H, Rino Y, Masuda M: Efficacy and tolerability of initial low-dose lenvatinib to treat differentiated thyroid cancer. Medicine (Baltimore) 98:e14774 (2019)

3. Matsuzu K, Sugino K, Masudo K, Nagahama M, Kitagawa W, Shibuya H, Ohkuwa K, Uruno T, Suzuki A, Magoshi S, Akaishi J, Masaki C, Kawano M, Suganuma N, Rino Y, Masuda M, Kameyama K, Takami H, Ito K: Thyroid lobectomy for papillary thyroid cancer: long-term follow-up study of 1,088 cases. World J Surg 38:68-79 (2014) 
4. Cabanillas ME, Habra MA: Lenvatinib: Role in thyroid cancer and other solid tumors. Cancer Treat Rev 42:47-55 (2016)

5. Durante C, Haddy N, Baudin E, Leboulleux S, Hartl D, Travagli JP, Caillou B, Ricard M, Lumbroso JD, De Vathaire F, Schlumberger M: Long-term outcome of 444 patients with distant metastases from papillary and follicular thyroid carcinoma: benefits and limits of radioiodine therapy. $J$ Clin Endocrinol Metab 91:2892-9 (2006)

6. Shaha AR, Migliacci JC, Nixon IJ, Wang LY, Wong RJ, Morris LGT, Patel SG, Shah JP, Tuttle RM, Ganly I: Stage migration with the new American Joint Committee on Cancer (AJCC) staging system (8th edition) for differentiated thyroid cancer. Surgery 165:6-11 (2019)

7. Nixon IJ, Wang LY, Migliacci JC, Eskander A, Campbell MJ, Aniss A, Morris L, Vaisman F, Corbo R, Momesso D, Vaisman M, Carvalho A, Learoyd D, Leslie WD, Nason RW, Kuk D, Wreesmann V, Morris L, Palmer FL, Ganly I, Patel SG, Singh B, Tuttle RM, Shaha AR, Gönen M, Pathak KA, Shen WT, Sywak M, Kowalski L, Freeman J, Perrier N, Shah JP: An International Multi-Institutional Validation of Age 55 Years as a Cutoff for Risk Stratification in the AJCC/UICC Staging System for Well-Differentiated Thyroid Cancer. Thyroid 26:373-80 (2016)

8. Sugino K, Nagahama M, Kitagawa W, Ohkuwa K, Uruno T, Matsuzu K, Suzuki A, Tomoda C, Hames KY, Akaishi J, Masaki C, Ito K: Distant Metastasis in Pediatric and Adolescent Differentiated Thyroid Cancer: Clinical Outcomes and Risk Factor Analyses. J Clin Endocrinol Metab 105(11):dgaa545 (2020)

9. Cottreau CM, Dashevsky I, Andrade SE, Li DK, Nekhlyudov L, Raebel MA, Ritzwoller DP, Partridge AH, Pawloski PA, Toh S: Pregnancy-Associated Cancer: A U.S. Population-Based Study. J Womens Health (Larchmt) 28:250-257 (2019)

10. Derwahl M, Nicula D: Estrogen and its role in thyroid cancer. Endocr Relat Cancer 21:T273-83 (2014)

11. Ito Y, Miyauchi A, Kudo T, Ota H, Yoshioka K, Oda H, Sasai H, Nakayama A, Yabuta T, Masuoka H, Fukushima M, Higashiyama T, Kihara M, Kobayashi K, Miya A: Effects of Pregnancy on Papillary Microcarcinomas of the Thyroid Re-Evaluated in the Entire Patient Series at Kuma Hospital. Thyroid 26:156-60 (2016)

12. Chen AC, Livhits MJ, Du L, Wu JX, Kuo EJ, Yeh MW, Leung AM: Recent Pregnancy Is Not Associated with High-Risk Pathological Features of Well-Differentiated Thyroid Cancer. Thyroid 28:68-71 (2018)

13. Xi C, Zhang Q, Song HJ, Shen CT, Zhang GQ, Sun JW, Qiu ZL, Luo QY: Pregnancy does not affect the Prognoses of Differentiated Thyroid Cancer Patients with Lung Metastases. J Clin Endocrinol Metab 106:e3185-e3197 (2021)

14. Takahashi S, Horie A, Yamamura S, Kawamura A, Yamaguchi A, Sunada M, Tani H, Mogami H, Hamanishi J, Kondoh E, Mandai M: Oncofertility care in young women and the outcomes of pregnancy 
over the last 5 years. Future Sci OA 7:FSO680 (2021)

15. Sullivan E, Wang Y, Chapman M, Chambers G: Success rates and cost of a live birth following fresh assisted reproduction treatment in women aged 45 years and older, Australia 2002-2004. Hum Reprod 23:1639-43 (2008)

16. Kanda Y: Investigation of the freely available easy-to-use software 'EZR' for medical statistics. Bone Marrow Transplant 48:452-8 (2013)

17. Ito $\mathrm{Y}$, Onoda N, Okamoto $\mathrm{T}$ : The revised clinical practice guidelines on the management of thyroid tumors by the Japan Associations of Endocrine Surgeons: Core questions and recommendations for treatments of thyroid cancer. Endocr J 67:669-717 (2020)

18. Song HJ, Qiu ZL, Shen CT, Wei WJ, Luo QY: Pulmonary metastases in differentiated thyroid cancer: efficacy of radioiodine therapy and prognostic factors. Eur J Endocrinol 173:399-408 (2015)

19. Sawka AM, Lakra DC, Lea J, Alshehri B, Tsang RW, Brierley JD, Straus S, Thabane L, Gafni A, Ezzat S, George SR, Goldstein DP: A systematic review examining the effects of therapeutic radioactive iodine on ovarian function and future pregnancy in female thyroid cancer survivors. Clin Endocrinol (Oxf) 69:479-90 (2008)

20. Nies M, Cantineau AEP, Arts EGJM, van den Berg MH, van Leeuwen FE, Muller Kobold AC, Klein Hesselink MS, Burgerhof JGM, Brouwers AH, van Dam EWCM, Havekes B, van den Heuvel-Eibrink MM, Corssmit EPM, Kremer LCM, Netea-Maier RT, van der Pal HJH, Peeters RP, Plukker JTM, Ronckers CM, van Santen HM, van der Horst-Schrivers ANA, Tissing WJE, Bocca G, van Dulmen-den Broeder E, Links TP: Long-Term Effects of Radioiodine Treatment on Female Fertility in Survivors of Childhood Differentiated Thyroid Carcinoma. Thyroid 30:1169-1176 (2020)

21. Ko KY, Yen RF, Lin CL, Cheng MF, Huang WS, Kao CH: Pregnancy Outcome After I-131 Therapy for Patients With Thyroid Cancer: A Nationwide Population-Based Cohort Study. Medicine (Baltimore) 95:e2685 (2016)

22. Wu JX, Young S, Ro K, Li N, Leung AM, Chiu HK, Harari A, Yeh MW: Reproductive outcomes and nononcologic complications after radioactive iodine ablation for well-differentiated thyroid cancer. Thyroid 25:133-8 (2015)

23. Sugiura-Ogasawara M, Ozaki Y, Kitaori T, Suzumori N, Obayashi S, Suzuki S: Live birth rate according to maternal age and previous number of recurrent miscarriages. Am J Reprod Immunol 62:314-9 (2009)

24. Kawase K, Nomura K, Nomura S, Akashi-Tanaka S, Ogawa T, Shibasaki I, Shimada M, Taguchi T, Takeshita E, Tomizawa Y, Hanazaki K, Hanashi T, Yamauchi H, Yamashita H, Nakamura S: How pregnancy and childbirth affect the working conditions and careers of women surgeons in Japan: findings of a nationwide survey conducted by the Japan Surgical Society. Surg Today 51:309-321 (2021) 

pregnancy on prognosis of differentiated thyroid cancer: clinical and molecular features. Eur $\mathrm{J}$ Endocrinol 170:659-66 (2014)

26. Harada M, Osuga Y: Fertility preservation for female cancer patients. Int J Clin Oncol 24:28-33 (2019)

\section{Figures}

Figure 1.

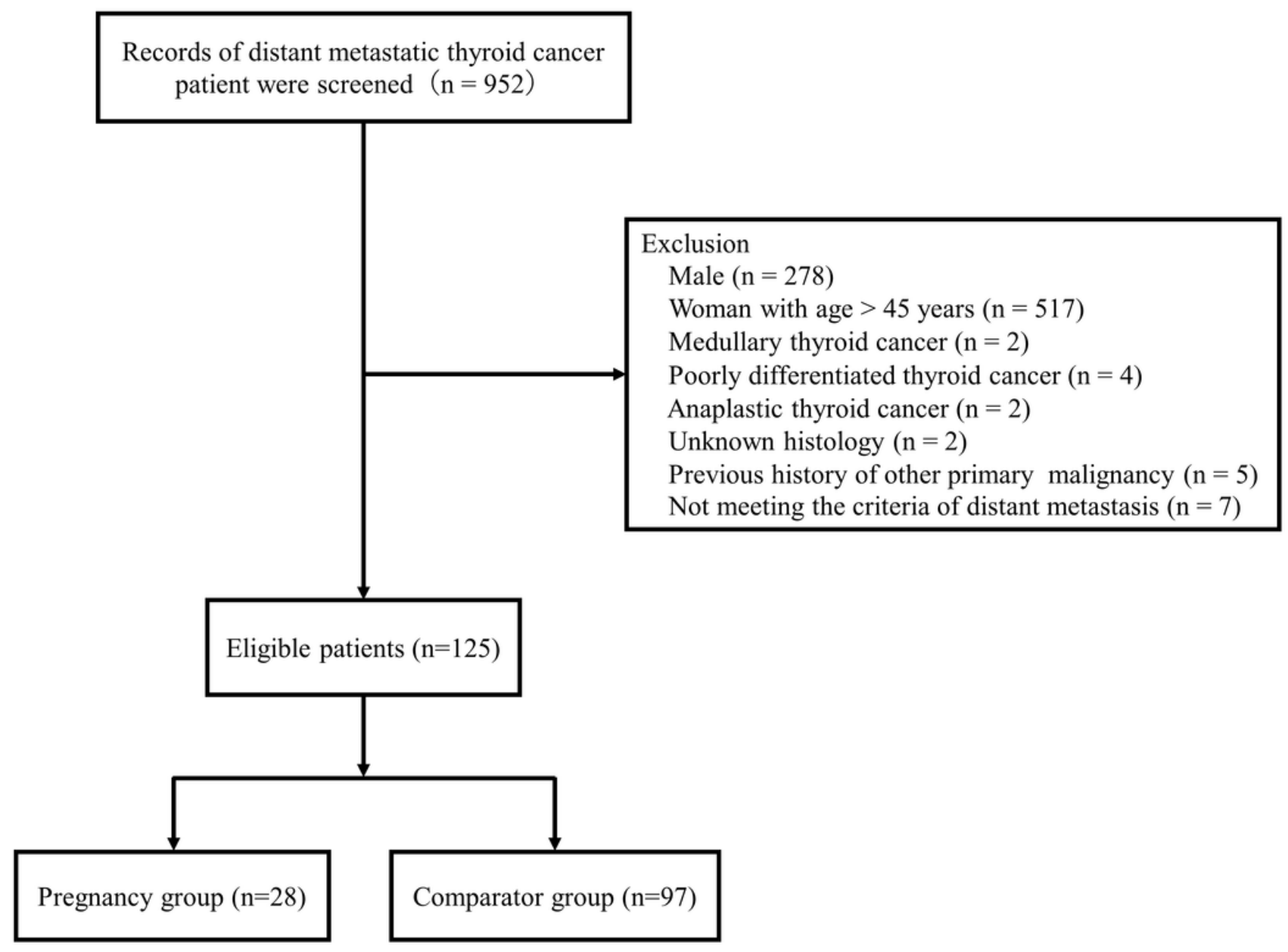

Figure 1

Enrollment and participation flow diagram. 
Figure 2.

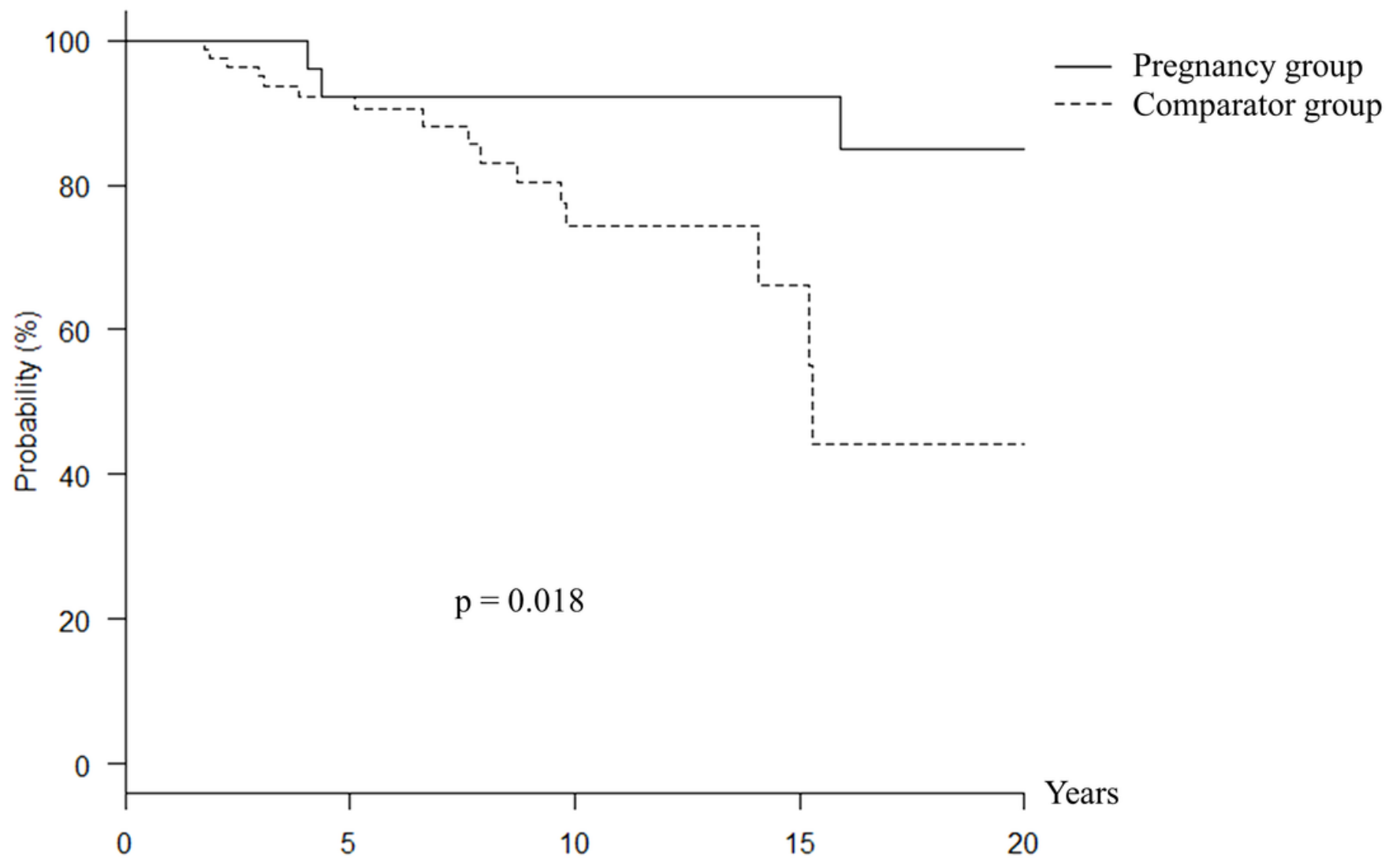

Figure 2

Progression-free survival. The 10-year progression free survival rates of pregnancy and comparator were $92.1 \%$ and $74.4 \%$, respectively $(p=0.018)$. 